\title{
Heat-kernel expansion at finite temperature
}

\author{
H. Boschi-Filho* and C. P. Natividade \\ Instituto de Física, Universidade Federal do Rio de Janeiro, RJ 21945, Caixa Postal 68528, Rio de Janeiro, Brazil \\ C. Farina ${ }^{\dagger}$ \\ Departamento de Física Teorica, Universidad de Zaragoza, 50009 Zaragoza, Spain
}

(Received 7 May 1991)

\begin{abstract}
We show how the zero-temperature result for the heat-kernel asymptotic expansion can be generalized to the finite-temperature one. We observe that this general result depends on the interesting ratio $\sqrt{\tau} / \beta$, where $\tau$ is the regularization parameter and $\beta=1 / T$, so that the zero-temperature limit $\beta \rightarrow \infty$ corresponds to the "cutoff" limit $\tau \rightarrow 0$. As an example, we discuss some aspects of the axial model at finite temperature.

PACS number(s): 11.10.Jj, 11.30.Rd
\end{abstract}

\section{INTRODUCTION}

One of the most important motivations for studying quantum field theories (QFT's) at nonzero temperature is closely related to the study of cosmology. Standard cosmology, or more generally, any reasonable cosmological model, predicts a very high temperature for matter and radiation in the early days of the Universe. We have no reason to expect that the symmetries present in the early Universe have remained the same ones until the present stage of the Universe, after cooling down for such a long period. Phase transitions certainly occurred and it is precisely their existence that justifies the study of QFT at finite temperature. Since the mass scale introduced by grand unified theories (GUT's) is inaccessible, the different cosmological predictions (which presumably involve temperature effects) stated by different theories may shed some light in the choice of the theory which better describes nature. Of course there are other branches of physics where temperature effects may have an important role, as for example, in heavy-ion collisions.

However, in this paper we shall not be concerned with early Universe calculations, or with any direct (detectable) temperature calculation, but with more technical aspects, regarding the formalism of QFT at finite temperature itself.

Dolan and Jackiw [1] have investigated the symmetry behavior of field theories at finite temperature. They showed, for instance, that in massless quantum electrodynamics in two dimensions the mass of the gauge boson of the theory ("photon mass") acquired dynamically remains the same for all finite temperatures. Their strategy was to show that the vacuum-polarization tensor at finite temperature coincides with the one at zero temperature.

*Permanent address: Departamento de Física e Química, Universidade Estadual Paulista, Campus de Guaratinguetá, CxP 205, 12500 Guaratinguetá, SP, Brazil.

†On leave from Instituto de Física, Universidade Federal do Rio de Janeiro, RJ, Brazil.
Recently, these results were rederived in the pathintegral framework by Reuter and Dittrich [2]. These authors used a technique very similar to Fujikawa's original work [3], where the chiral anomaly is extracted from the noninvariance of the fermionic functional measure under infinitesimal chiral transformations. In fact, in order to obtain a regularized expression for the fermionic determinant at finite temperature, Reuter and Dittrich used the $\zeta$-function regularization prescription, introduced a long time ago by some authors [4]. This technique proved to be a very useful and elegant method for computing anomalies in QFT [5] (an excellent review of this method containing as applications the computation of anomalies as well as the exact solution of several twodimensional models is given by Gamboa-Saraví et al. [6]).

Motivated by the paper of Reuter and Dittrich [2], our purpose here is to analyze in a more systematic way what happens to the asymptotic expansion of the heat kernel when we deal with QFT at finite temperature using the path-integral Euclidean space-time formalism [7]. We construct a generalization of this asymptotic expansion at finite temperature trying to keep as much as possible a close analogy with the zero-temperature case. We also define generalized Seeley's coefficients, which are now $\beta$ dependent (as well as dependent on the regularization parameter $\tau$ ), in a natural way to give us a transparent interpretation of the limits $\beta \rightarrow \infty$ and $\tau \rightarrow 0$. As will become clear, this expansion is of fundamental importance in the evaluation of the Seeley's coefficients [8] through the $\zeta$-function method.

This work is organized as follows. In Sec. II, we review some aspects of the heat-kernel asymptotic expansion at zero temperature. In Sec. III, we obtain the main results of this paper. Here, we construct explicitly the expression for the diagonal part of the heat kernel at finite temperature. As an application, we discuss in Sec. IV the axial model at finite temperature and derive its anomaly. This model was introduced by Rothe and Stamatescu [9], and since then, it has been discussed at zero temperature in a flat space-time [10], as well as in a curved space-time [11]. Here, in order to consider this 
model at nonzero temperature, we calculate the Jacobian associated with infinitesimal chiral transformations over the fermionic functional measure at finite temperature. Section $V$ is left for the conclusions and some final remarks. An appendix is included to make this paper selfcontained.

\section{THE HEAT-KERNEL EXPANSION AT ZERO TEMPERATURE}

In this section, we review for the sake of completeness the heat-kernel asymptotic expansion at zero temperature. It was originally constructed by DeWitt [12] in curved space-times based on the Schwinger integral representation for propagators [13], so this is also known as the Schwinger-DeWitt expansion. To solve the integral equation for the coefficients, DeWitt used recursive relations, but we will follow the constructive approach of Nepomechie [14], which is better suited for the pathintegral treatment since it is related to Fujikawa's method, although restricted to flat space-time.

It is well known that in nonperturbative calculations of chiral anomalies using a path-integral approach, we must regularize the ill-defined quantity [3] (the "anomaly")

$$
\mathcal{A} \equiv \sum_{n} \phi_{n}^{\dagger}(x) \gamma_{5} \phi_{n}(x)
$$

where $\left\{\phi_{n}(x)\right\}$ is usually the set of eigenfunctions of the Hermitian Dirac operator $\not D$ present in the original Lagrangian.

There are different ways of regularizing the last expression. For instance, one can use the heat-kernel method (this is essentially Fujikawa's approach [3]), and write the regularized anomaly as

$$
\left.\mathcal{A}\right|_{\mathrm{HK}}=\lim _{\tau \rightarrow 0} \operatorname{tr}\left[\gamma_{5} H(x, x ; \tau)\right]
$$

where

$$
\begin{aligned}
H\left(x, x^{\prime} ; \tau\right) & =\langle x| e^{-\tau D^{2}\left|x^{\prime}\right\rangle} \\
& =e^{-\tau D_{x}^{2}} \delta\left(x-x^{\prime}\right) .
\end{aligned}
$$

Alternatively, one can use the $\zeta$-function approach and write [6]

$$
\left.\mathcal{A}\right|_{\zeta}=\lim _{s \rightarrow 0} \operatorname{tr}\left[\gamma_{5} K(x, x ; s)\right]
$$

where

$$
\begin{aligned}
K\left(x, x^{\prime} ; s\right) & =\left\langle x\left|\left(\not D^{2}\right)^{-s}\right| x^{\prime}\right\rangle \\
& =\left(\not D_{x}^{2}\right)^{-s} \delta\left(x-x^{\prime}\right) .
\end{aligned}
$$

It is called sometimes the negative-power kernel and it is related to the heat kernel by a Mellin transformation

$$
K(x, x ; s)=\frac{1}{\Gamma(s)} \int_{0}^{\infty} \tau^{s-1} H(x, x ; \tau) d \tau .
$$

It is worth mentioning that the heat-kernel approach does not always yield finite results. This accidentally happens in the chiral-anomaly case due to the presence of the $\gamma_{5}$ matrix (and a trace to be performed), but divergent results survive when this method is used, for instance, in the computation of the conformal anomaly. On the other hand, the $\zeta$-function approach always gives finite results. A comparison of these methods with explicit examples can be found in the literature [15]. In any case, an asymptotic expansion $(\tau<<1)$ for the heat kernel is needed. Let us, then, show how to obtain this asymptotic expansion for a general case. We shall follow Nepomechie's construction [14].

Consider the Euclidean space operator $\Delta$, which in coordinate space is given by

$$
\Delta_{x}=-D_{\mu} D_{\mu}+X,
$$

where

$$
D_{\mu}=\partial_{\mu}+A_{\mu},
$$

and we shall be restricted by the condition that $A_{\mu}$ and $X$ are not differential operators. Then, we define the heat kernel in a flat $d$-dimensional space-time associated with the operator $\Delta$ by

$$
\begin{aligned}
H\left(x, x^{\prime} ; \tau\right) & =\left\langle x\left|e^{-\tau \Delta}\right| x^{\prime}\right\rangle \\
& =e^{-\tau \Delta x} \delta^{d}\left(x-x^{\prime}\right) .
\end{aligned}
$$

It can be shown that the kernel (2.9) satisfies the heat equation

$$
\left[\frac{\partial}{\partial \tau}+\Delta_{x}\right] H\left(x, x^{\prime} ; \tau\right)=0
$$

After using the Fourier representation for the Dirac delta function and also making a careful use of some operator identities, the diagonal part of the heat kernel (2.9) can be written as [14]

$$
\begin{aligned}
H(x, x ; \tau)= & {\left[\frac{1}{4 \pi \tau}\right]^{d / 2} } \\
& \times \int \frac{d^{d} k}{\pi^{d / 2}} e^{-k^{2}} \sum_{m=0}^{\infty} \frac{1}{m !}\left(2 i k \cdot D \sqrt{\tau}-\tau \Delta_{x}\right)^{m}
\end{aligned}
$$

Now we can expand the binomial as

$$
\left(2 i k \cdot D \sqrt{\tau}-\tau \Delta_{x}\right)^{m}=\sum_{j=0}^{m} \sum_{\text {cyc. perm. }}(-1)^{j}(2 i k \cdot D)^{j}\left(\Delta_{x}\right)^{m-j} \tau^{m-j / 2}
$$

where the sum over cyclic permutations of the noncommuting factors $k \cdot D$ and $\Delta_{x}$ is responsible for the absence of the usual binomial coefficients. Noting that all terms containing odd powers of $k_{\mu}$ in (2.11) vanish (due to integration over $k$ ), we conclude that only the integer powers of $\tau$ do contribute to the asymptotic expansion of the heat kernel 


$$
H(x, x ; \tau)=\left(\frac{1}{4 \pi \tau}\right]^{d / 2} \sum_{n=0}^{\infty} \tau^{n} \sum_{l=0}^{n} \frac{1}{(l+m) !} \int \frac{d^{d} k}{\pi^{d / 2}} e^{-k^{2}} \sum_{\text {cyc. perm. }}(2 i k \cdot D)^{2 l}\left(\Delta_{x}\right)^{n-l}
$$

where we have defined $n=m-j / 2$ and $l=m-n$.

Therefore, the asymptotic expansion for the heat kernel reads

$$
H(x, x ; \tau)=\left(\frac{1}{4 \pi \tau}\right)^{d / 2} \sum_{m=0}^{\infty} a_{m}(x) \tau^{m}
$$

where the Seeley's coefficients are identified as

$a_{n}=\sum_{l=0}^{n} \frac{1}{(l+n) !} \int \frac{d^{d} k}{\pi^{d / 2}} e^{-k^{2}} \sum_{\text {cyc. perm. }}(2 i k \cdot D)^{2 l}\left(\Delta_{x}\right)^{n-l}$.

As it is well known in the literature, these coefficients do not depend on the dimension $d$ of the space-time. Here this can be understood if we realize that all dependence on $d$ will disappear after the following integrations are done:

$$
\begin{aligned}
& \int \frac{d^{d} k}{\pi^{d / 2}} e^{-k^{2}}=1 \\
& \int \frac{d^{d} k}{\pi^{d / 2}} e^{-k^{2} k_{\mu} k_{v}=\delta_{\mu \nu}} \\
& \int \frac{d^{d} k}{\pi^{d / 2}} e^{-k^{2}} k_{\mu} k_{v} k_{\alpha} k_{\beta}=\frac{1}{4}\left(\delta_{\mu \nu} \delta_{\beta \alpha}+\delta_{\mu \alpha} \delta_{\nu \beta}+\delta_{\mu \beta} \delta_{v \alpha}\right)
\end{aligned}
$$

Using (2.15) and (2.16) we can calculate, for example, the first three coefficients

$$
\begin{aligned}
& a_{0}=1, \\
& a_{1}=-X \\
& a_{2}=\frac{1}{2} X^{2}-\frac{1}{6}\left(D^{2} X\right)+\frac{1}{12}\left[D_{\mu}, D_{v}\right]^{2} .
\end{aligned}
$$

The first one reflects a normalization choice and the last two are useful to calculate the chiral anomalies in twoand four-dimensional space-times, respectively [6].

\section{THE HEAT-KERNEL EXPANSION AT FINITE TEMPERATURE}

The results of the previous section can be carefully extended to the finite-temperature case. The main difference is that the space-time symmetry is broken in the sense that the limit of the integration on the time variable in the partition function is restricted to the interval $(0, \beta=1 / T)$. In all subsequent calculations thermal equilibrium is assumed. Then, thermal Green's functions are simply given by

$$
\begin{aligned}
G_{\beta}\left(\Phi\left(x_{1}\right) \Phi\left(x_{2}\right) \cdots \Phi\left(x_{m}\right)\right) & =\frac{\operatorname{tr}\left[e^{-\beta \mathcal{H}} T\left(\Phi\left(x_{1}\right) \Phi\left(x_{2}\right) \cdots \Phi\left(x_{m}\right)\right)\right]}{\operatorname{tr}\left(e^{-\beta \mathcal{H}}\right)} \\
& =\frac{\sum_{n} e^{-\beta E_{n}}\left\langle n\left|T\left(\Phi\left(x_{1}\right) \Phi\left(x_{2}\right) \cdots \Phi\left(x_{m}\right)\right)\right| n\right\rangle}{\sum_{n} e^{-\beta E_{n}}},
\end{aligned}
$$

where $\{|n\rangle\}$ is a complete set of eigenfunctions of $\mathcal{H}$ and $T$ means the usual time-ordering operator. The zerotemperature limit can be straightforwardly obtained from (3.1).

In analogy with the zero-temperature case, a thermal generating functional can also be constructed,

$$
Z_{\beta}[j]=\frac{\operatorname{tr}\left\{e^{-\beta \mathcal{H}} T\left[\exp \left[i \int j(x) \Phi(x) d x\right]\right]\right\}}{\operatorname{tr}\left(e^{-\beta \mathcal{H}}\right)},
$$

where the trace is over any complete set of states. Hence, making use of a perturbative scheme, we can compute, in principle, any quantity which is calculable at zero temperature.

It is worth mentioning that, contrary to what happens at zero temperature, to work with the Minkowski or Eu- clidean theory makes a great difference, and distinct techniques have been constructed for these two cases. A very clear discussion of both methods (imaginary time and real time), is given by Rivers [16]. We shall consider here the Euclidean-time formalism [7].

However, in our following discussion, the thermal generating functional is not really necessary. It suffices to consider the partition function $Z(\beta)$ of the theory at hand.

It can be shown that $Z(\beta)$ for quantum electrodynamics is given by [2] (from now on we shall work in a $d$ dimensional space-time)

$$
\begin{aligned}
Z(\beta)=\int_{F_{\beta}} & {\left[d A_{\mu}\right][d \psi][d \bar{\psi}] } \\
& \times \exp \left[\int_{0}^{\beta} d x_{0} \int d x_{i}\left[\bar{\psi}(i \not D-m) \psi-\frac{1}{4} F_{\mu \nu}^{2}\right]\right),
\end{aligned}
$$


where $\not D \equiv i \gamma^{\mu}\left(\partial_{\mu}-e A_{\mu}\right)$ and the subscript $F_{\beta}$ means that the functional integration must be taken over those functions which are periodic (for Bose fields) and antiperiodic (for Fermi fields) under translation $x_{0} \rightarrow x_{0}+\beta$.

The heat-kernel expansion appears when we regularize the anomaly in order to compute the Jacobian associated with infinitesimal chiral rotations of the fermionic fields. In the above case, the heat kernel would be

$$
H_{\beta}\left(x, x^{\prime} ; \tau\right)=e^{-\tau \Delta_{x}} \delta_{\beta}^{d}\left(x-x^{\prime}\right),
$$

with $\Delta_{x}=\not D^{2}+m^{2}$ and the subscript $\beta$ in the Dirac delta function is a reminder that its Fourier representation must be compatible with the periodic (antiperiodic) conditions on the variable $x_{0}$.

Observing that $\delta_{\beta}^{d}\left(x-x^{\prime}\right)$ appears through the completeness relation of the eigenfunctions of $\not D$, and remembering that this operator acts on antiperiodic functions, the Fourier representation for the Dirac delta function takes the form

$$
\begin{aligned}
\delta_{\beta}^{d}\left(x-x^{\prime}\right) & =\delta^{d-1}\left(\mathbf{x}-\mathbf{x}^{\prime}\right) \frac{1}{\beta} \sum_{n=-\infty}^{\infty} e^{(2 i \pi / \beta)(n+1 / 2)\left(x_{0}-x_{0}^{\prime}\right)} \\
& =\sum_{n=-\infty}^{+\infty} \int \frac{d^{d} k}{(2 \pi)^{d / 2}} e^{i k\left(x-x^{\prime}\right)}\left(\frac{2 \pi}{\beta}\right] \delta\left[k_{0}-\frac{2 \pi}{\beta}\left(n+\frac{1}{2}\right)\right] .
\end{aligned}
$$

If one is interested in temperature effects on boson fields, $(n+1 / 2)$ must be replaced by $n$ in the above formulas because of the periodic behavior of these fields under the translation $x_{0} \rightarrow x_{0}+\beta$.

Using (3.5) we can extend the zero-temperature definition of heat kernel (2.3) to the finite-temperature case:

$$
H_{\beta}\left(x, x^{\prime} ; \tau\right)=e^{-\tau \Delta_{x}} \sum_{n=-\infty}^{+\infty} \int \frac{d^{d} k}{(2 \pi)^{d / 2}} e^{i k\left(x-x^{\prime}\right)}\left(\frac{2 \pi}{\beta}\right] \delta\left[k_{0}-\frac{2 \pi}{\beta}\left(n+\frac{1}{2}\right)\right] \text {. }
$$

Proceeding with calculations similar to those for the zero-temperature case, we find that the diagonal part of $H_{\beta}\left(x, x^{\prime} ; \tau\right)$ is given by

$$
H_{\beta}(x, x ; \tau)=\left(\frac{1}{4 \pi \tau}\right)^{d / 2} \sum_{n=-\infty}^{+\infty}\left[\frac{2 \pi \sqrt{\tau}}{\beta}\right] \int \frac{d^{d} k}{\pi^{d / 2}} \delta\left[k_{0}-\frac{2 \pi}{\beta} \sqrt{\tau}\left(n+\frac{1}{2}\right)\right] e^{-k^{2}} \sum_{m=0}^{\infty} \frac{1}{m !}\left(2 i k \cdot D \sqrt{\tau}-\tau \Delta_{x}\right)^{m},
$$

where we have made the usual rescaling [14] $k_{\mu} \rightarrow k_{\mu} / \sqrt{\tau}$. Performing the integration over $k_{0}$ and expanding the binomial, we find

$$
\begin{aligned}
H_{\beta}(x, x ; \tau)= & \left(\frac{1}{4 \pi \tau}\right]^{d / 2}\left[\frac{4 \pi \tau}{\beta^{2}}\right]^{1 / 2} \\
& \times \int \frac{d^{d-1} k_{j}}{\pi^{(d-1) / 2}} \sum_{n=-\infty}^{+\infty} \exp \left[\frac{-4 \pi^{2} \tau}{\beta^{2}}(n+1 / 2)^{2}-k_{j}^{2}\right] \\
& \times\left.\sum_{m=0}^{\infty} \sum_{l=0}^{m} \sum_{\text {cyc. perm. }} \frac{(-1)^{l}}{(m+l) !}(2 k \cdot D)^{2 l}\left(\Delta_{x}\right)^{m-l} \tau^{m}\right|_{k_{0}=(2 \pi / \beta) \sqrt{\tau}(n+1 / 2)},
\end{aligned}
$$

where we have dropped the half-integer powers of $\tau$ as in the zero-temperature case since the integrals over odd powers of $k_{j}$ still vanish and the sum over odd powers of $k_{0}$ with respect to $n$, vanishes too.

In order to keep the analogy with the zero-temperature case, and also for future convenience when analyzing the limits of physical interest, we shall cast $H_{\beta}(x, x ; \tau)$ into the form

$$
H_{\beta}(x, x ; \tau)=\left(\frac{1}{4 \pi \tau}\right)^{d / 2} \sum_{m=0}^{\infty} a_{m}(\sqrt{\tau} / \beta) \tau^{m}
$$

but this time, as we will show explicitly, the "generalized coefficients" $a_{m}$ are functions of the ratio $\sqrt{\tau} / \beta$ that do not admit a power-series expansion at $\sqrt{\tau} / \beta=0$. Comparing (3.8) and (3.9), we see that these generalized coefficients are given by

$$
\begin{aligned}
a_{m}(\sqrt{\tau} / \beta)=\frac{2 \sqrt{\pi \tau}}{\beta} \int \frac{d^{d-1} k_{j}}{\pi^{(d-1) / 2}} \sum_{n=-\infty}^{+\infty} & \exp \left[\frac{-4 \pi^{2}}{\beta^{2}}(n+1 / 2)^{2} \tau-k_{j}^{2}\right] \\
& \times\left.\sum_{l=0}^{m} \sum_{\text {cyc. perm. }} \frac{(-1)^{l}}{(m+l) !}(2 k \cdot D)^{2 l}\left(\Delta_{x}\right)^{m-l} \tau^{m}\right|_{k_{0}=(2 \pi / \beta) \sqrt{\tau}(n+1 / 2)}
\end{aligned}
$$


The series over $n$ is slowly convergent but we can transform it into a rapidly convergent one in order to be able to take the first few terms as an approximate result. This can be done using a generalized theta function transformation [17] (see the Appendix)

$$
\sum_{n=-\infty}^{+\infty}\left[\gamma(n+1 / 2)^{2}\right]^{p} e^{-\gamma(n+1 / 2)^{2}}=\left(\frac{\pi}{\gamma}\right)^{1 / 2} \frac{(2 p-1) ! !}{2 p}\left[1+2 \sum_{n=1}^{\infty}(-1)^{n} e^{-\pi^{2} n^{2} / \gamma} \sum_{l=0}^{p}\left[\frac{-\pi^{2} n^{2}}{\gamma}\right]^{l} C^{p}\right],
$$

where

$$
C p=\frac{2^{l} p !}{l !(p-l) !(2 l-1) ! !}
$$

$p$ is a non-negative integer and $(2 p-1) ! !=1 \times 3 \times \cdots \times(2 p-1)$. From (3.10) we can calculate the temperaturedependent coefficients $a_{m}(\sqrt{\tau} / \beta)$. Using (2.16a) to perform integrations over $k_{j}$ we find

$$
a_{0}(\sqrt{\tau} / \beta)=2 \frac{\sqrt{\pi \tau}}{\beta} \sum_{n=-\infty}^{+\infty} \exp \left[\frac{-4 \pi^{2}}{\beta^{2}} \tau(n+1 / 2)^{2}\right) .
$$

Applying the transformation (3.11) [with $p=0$ and $(2 p-1) ! !=1]$ in $a_{0}(\sqrt{\tau} / \beta)$ we arrive at

$$
a_{0}(\sqrt{\tau} / \beta)=1+2 \sum_{n=1}^{\infty}(-1)^{n} \exp \left[\frac{-n^{2} \beta^{2}}{4 \tau}\right) \text {. }
$$

This generalized coefficient reduces to the zero-temperature one when we take the limit $\beta \rightarrow \infty$, so $a_{0}(0)=a_{0}$, given by Eq. (2.17a).

In order to compute $a_{1}(\sqrt{\tau} / \beta)$, we also start from (3.10). Doing the integrations $(2.16 \mathrm{a})$ and $(2.16 \mathrm{~b})$ and applying the transformation (3.11) with $p=0,1$ we get

$$
\begin{aligned}
a_{1}(\sqrt{\tau} / \beta) & =2 \frac{\sqrt{\pi \tau}}{\beta} \sum_{n=-\infty}^{+\infty} \exp \left[\frac{-4 \pi^{2}}{\beta^{2}} \tau(n+1 / 2)^{2}\right]\left[\Delta_{x}+D_{j}^{2}+2 D_{0}^{2}\left(\frac{4 \pi^{2}}{\beta^{2}}\right) \tau(n+1 / 2)^{2}\right] \\
& =-X\left[1+2 \sum_{n=1}^{\infty}(-1)^{n} \exp \left[\frac{-n^{2} \beta^{2}}{4 \tau}\right]\right]+D_{0}^{2} \sum_{n=1}^{\infty}(-1)^{n}\left(\frac{\beta^{2} n^{2}}{\tau}\right) \exp \left(\frac{-n^{2} \beta^{2}}{4 \tau}\right),
\end{aligned}
$$

where

$$
X=\Delta_{x}+D^{2} \text {. }
$$

The coefficient (3.15) also reduces to the zerotemperature one $a_{1}$, given by $(2.17 \mathrm{~b})$ in the limit $\beta \rightarrow \infty$. In fact, only the term multiplied by $X$ in (3.15) does not vanish. This happens because in these calculations, as well as in the zero-temperature one, we have assumed that the product

$$
e^{-i k \cdot x}\left(\tau \Delta_{x}\right) e^{i k \cdot x}
$$

which appears repeatedly in the power-series expansion of $\exp \left(-\tau \Delta_{x}\right)$ (after a convenient insertion of unities such as $1=e^{i k x} e^{-i k x}$ ) was applied to an arbitrary function $f(x)$ which, at the end of the calculations, must be put equal to one. This implies that any remainder differential operator from these produces must vanish, so we can write

$$
a_{1}(\sqrt{\tau} / \beta)=-X\left[1+2 \sum_{n=1}^{\infty}(-1)^{n} \exp \left(\frac{-n^{2} \beta^{2}}{4 \tau}\right)\right] .
$$

It is interesting to note that this final cancellation of the differential operators does not really occur in the zero-temperature case, because the space-time symmetry is not broken. The temperature-dependent behavior of the first coefficients $a_{0}(\sqrt{\tau} / \beta)$ and $a_{1}(\sqrt{\tau} / \beta)$ are also accomplished by the others of highest order. This is so because the temperature-independent terms [those which are not multiplied by $\left.\exp \left(-n^{2} \beta^{2} / 4 \tau\right)\right]$, and the terms which are only multiplied by $\exp \left(-n^{2} \beta^{2} / 4 \tau\right)$ without any power of $-n^{2} \beta^{2} / \tau$ can be collected together reproducing the zero-temperature coefficients times

$$
1+2 \sum_{n=1}^{\infty}(-1)^{n} \exp \left(\frac{-n^{2} \beta^{2}}{4 \tau}\right) \text {. }
$$

The remainders depend on $\exp \left(-n^{2} \beta^{2} / 4 \tau\right)$ times some power of $n^{2} \beta^{2} / \tau$ and also retain differential operators (even powers of $D_{0}$ ), so that they vanish at the end of the calculations. With this established, we write a general relation connecting the generalized coefficients $a_{m}(\sqrt{\tau} / \beta)$ with their zero-temperature partners $a_{m}(0)$ :

$a_{m}(\sqrt{\tau} / \beta)=a_{m}(0)\left[1+2 \sum_{n=1}^{\infty}(-1)^{n} \exp \left[\frac{-n^{2} \beta^{2}}{4 \tau}\right]\right]$.

Besides the practical motivation of this formula, we want to discuss its physical implications. First, the temperature dependence of the coefficients is exactly the 
same for all coefficients of any order, so that, temperature is a global effect. This homogeneous behavior, may wrongly suggest a renormalization in all coefficients, but this would depend itself on the temperature. Second, all temperature dependence appears through the ratio $\sqrt{\tau} / \beta$. Since the regularization prescribes that the limit $\tau \rightarrow 0$ must be taken, we see that this limit is equivalent to taking $\beta \rightarrow \infty$, so the complete regularization is temperature independent. This behavior explains why such quantities as anomalies which can be calculated from the coefficients $a_{m}(\sqrt{\tau} / \beta)$ are free from the temperature.

The interesting result given by (3.20) can also be extended to the heat-kernel expansion. Substituting (3.20) into (3.9) we obtain

$$
\begin{aligned}
H_{\beta}(x, x ; \tau)= & {\left[\frac{1}{4 \pi \tau}\right]^{d / 2} } \\
& \times \sum_{m=0}^{\infty} a_{m}(0) \tau^{m} \\
& \times\left[1+2 \sum_{n=1}^{\infty}(-1)^{n} e^{-n^{2} \beta^{2} / 4 \tau}\right] .
\end{aligned}
$$

Comparing this finite-temperature expansion with the zero-temperature one $(2.14)$ we can finally state that

$$
H_{\beta}(x, x ; \tau)=H(x, x ; \tau)\left(1+2 \sum_{n=1}^{\infty}(-1)^{n} e^{-n^{2} \beta^{2} / 4 \tau}\right) \text {. }
$$

Again the limits $\beta \rightarrow \infty$ and $\tau \rightarrow 0$ are equivalent. This fact shows that the finite-temperature expansion reduces to the zero-temperature one in an elegant and transparent way.

\section{AXIAL MODEL AT FINITE TEMPERATURE}

As we have mentioned before, even if we adopt the $\zeta$ function regularization approach to compute the anomaly, Seeley's coefficients, present in the heat-kernel asymptotic expansion, are needed. This fact has already been reported in the literature for zero-temperature calculations. In this section, we will show how to use the generalized heat-kernel expansion (3.21) together with a Mellin transformation in order to compute the anomaly through the $\xi$-function technique for theories at finite temperature. We shall work in a concrete example.

Let us consider the axial model at finite temperature. This model describes the interaction of a massive pseudoscalar field $\varphi$ with a massless fermion field $\psi$ in terms of a derivative coupling. The corresponding partition function for this model is given by [18]

$$
\begin{aligned}
Z(\beta)=N(\beta) \int[ & d \psi][d \bar{\psi}][d \varphi] \\
& \times \exp \left(-\int d^{2} x\left(\bar{\psi} \widetilde{D} \psi+i \mathcal{L}_{\varphi}\right)\right],
\end{aligned}
$$

where

$$
\widetilde{D}=\gamma^{\mu}\left(\partial_{\mu}+i g_{0} \gamma_{5} \partial_{\mu} \varphi\right)
$$

and

$$
\mathcal{L}_{\varphi}=\frac{1}{2}\left(\partial_{\mu} \varphi \partial^{\mu} \varphi-m_{0}^{2} \varphi^{2}\right) .
$$

In fact, only the fermionic sector of $Z(\beta)$ will be relevant for the discussion regarding the anomaly. Following the standard procedure [3], we make the following infinitesimal chiral transformations

$$
\begin{aligned}
& \psi=\left[1+i g_{0} \gamma_{5} \alpha(x)\right] \psi^{\prime}, \\
& \bar{\psi}=\bar{\psi}^{\prime}\left[1+i g_{0} \gamma_{5} \alpha(x)\right] .
\end{aligned}
$$

Transformations (4.4) give rise, as usual, to the nontrivial Jacobian

$$
J(\alpha)=\exp \left[2 i g_{0} \int d^{2} x \alpha(x) \mathcal{A}(x)\right],
$$

where, as before, $\mathcal{A}(x)=\Sigma_{m} \phi_{m}^{\dagger} \gamma_{5} \phi_{m}$, and the factor 2 is due to both contributions, one coming from the change of $[d \psi]$ and the other from $[d \bar{\psi}]$.

At finite temperature, the $\zeta$-function regularization prescription means to write

$$
\left.\mathcal{A}(x)\right|_{\zeta}=\lim _{s \rightarrow 0} \operatorname{tr}\left[\gamma_{5} K^{\beta}(x, x ; s)\right],
$$

where

$$
K^{\beta}(x, x ; s)=\frac{1}{\Gamma(s)} \int_{0}^{\infty} d \tau \tau^{s-1} H_{\beta}(x, x ; \tau),
$$

with $H^{\beta}(x, x ; \tau)$ given by the generalized expansion (3.21)

Substituting these expressions and splitting the integral limits in (4.7) in order to justify the use of the asymptotic expansion [19], we have (observe that now $d=2$ )

$$
\begin{aligned}
K^{\beta}(x, x ; s)= & \frac{1}{4 \pi \Gamma(s)} \int_{0}^{\delta} d \tau \tau^{s-2} \sum_{m=0}^{\infty} a_{m}(\sqrt{\tau} / \beta) \tau^{m} \\
& +\frac{1}{\Gamma(s)} \int_{\delta}^{\infty} d \tau \tau^{s-1} H_{\beta}(x, x ; \tau)
\end{aligned}
$$

where $\delta$ is an arbitrary small parameter.

Since $\left.H_{\beta}(x, x ; \tau)\right|_{\tau \rightarrow 0} \rightarrow \exp \left(-\lambda_{0} \tau\right)$ (we are assuming positive-definite operators), the second integral in (4.8) is some entire analytical function of $s$ in the whole complex plane, say $F(s)$.

On the other hand, we have that the first integral is equal to

$$
\begin{aligned}
& \frac{1}{4 \pi \Gamma(s)} \int_{0}^{\delta} d \tau \tau^{s-2} \sum_{m=0}^{\infty} a_{m}(0) \tau^{m} \\
&+\frac{1}{2 \pi \Gamma(s)} \int_{0}^{\delta} d \tau \tau^{s-2} \sum_{m=0}^{\infty} a_{m}(0) \tau^{m} \\
& \quad \times \sum_{n=1}^{\infty}(-1)^{n} \exp \left(\frac{-n^{2} \beta^{2}}{4 \tau}\right) .
\end{aligned}
$$

The first integral in the above expression leads to the usual result for $K^{(0)}(x, x ; s)$ at zero temperature. It is easy to see that the second integral in this expression vanishes in the "splitting limit" $\delta \rightarrow 0$. Substituting this result in (4.7), collecting the powers in $\tau$, and performing the integration, we obtain 


$$
\begin{aligned}
K^{\beta}(x, x ; s)= & \frac{s}{4 \pi \Gamma(s+1)} \sum_{m=0}^{\infty} a_{m}(0) \frac{\delta^{s+m-1}}{s+m-1} \\
& +\frac{s F(s)}{\Gamma(s+1)} .
\end{aligned}
$$

Taking the limit $s \rightarrow 0$ in the above expression we see that only one term of the series will contribute, that one for which $m-1=0$. Consequently,

$$
\lim _{s \rightarrow 0} K^{\beta}(x, x ; s)=\frac{1}{4 \pi} a_{1}(0) .
$$

Using (2.17b) and the fact that here $X=-\frac{1}{2} i g_{0} \gamma_{5} \epsilon^{\mu v} F_{\mu \nu}^{5}$, we have

$$
\lim _{s \rightarrow 0} K^{\beta}(x, x ; s)=\frac{1}{8 \pi}\left(i g_{0} \gamma_{5} \epsilon^{\mu v} F_{\mu v}^{5}\right),
$$

where $F_{\mu \nu}^{5} \equiv \partial_{\mu} A_{v}^{5}-\partial_{v} A_{\mu}^{5}$, with $A_{\mu}^{5} \equiv \epsilon^{\mu v} \partial_{\nu} \varphi$. Hence

$$
\left.2 \mathcal{A}(x)\right|_{\zeta}=\frac{1}{2 \pi} \operatorname{tr}\left[\gamma_{5} a_{1}(0)\right]=\frac{i g_{0}}{2 \pi} \epsilon^{\mu v} F_{\mu v}^{5}
$$

and we find the regularized Jacobian (after coming back to the original $\varphi$ )

$$
\begin{aligned}
J_{\beta}(\alpha) & =\exp \left[\frac{-i g_{0}^{2}}{2 \pi} \int d^{2} x \alpha(x) \epsilon^{\mu v} F_{\mu \nu}^{5}\right] \\
& =\exp \left[\frac{-g_{0}^{2}}{2 \pi} \int d^{2} x \alpha(x) \partial_{\mu} \varphi \partial^{\mu} \varphi\right) .
\end{aligned}
$$

In order to decouple the fermion field from the pseudoscalar one, we can proceed in exactly the same way as in the zero-temperature case, that is, we must make a finite (and not infinitesimal) chiral transformation with $\alpha(x)=\varphi(x)$ and now compute the corresponding nontrivial Jacobian arising from this transformation. Although this is not an easy task, the result can be obtained by any iterative procedure such as those exposed in Refs. $[3,6]$. For the model at hand, it can be shown straightforwardly that the final effective action reads

$S_{\mathrm{eff}}=\int d^{2} x\left[\frac{1}{2}\left[1-\frac{g_{0}^{2}}{2 \pi}\right] \partial_{\mu} \varphi \partial^{\mu} \varphi-\frac{m_{0}^{2}}{2} \varphi^{2}+i \bar{\chi} \partial_{\mu} \chi\right]$

where $\chi$ is a free fermion field exactly as occurs at zero temperature. This effective action leads to the following equation of motion for the $\varphi$ field

$$
\left(\partial^{2}+m_{R}^{2}\right) \varphi=0,
$$

where

$$
m_{R}^{2}=\frac{m_{0}^{2}}{\left(1-g_{0}^{2} / 2 \pi\right)}
$$

is, then, identified with the renormalized mass of the theory and coincides precisely with that found in the zero-temperature case. This was an expected result, since the (finite) mass renormalization present in this model is due to the anomalous divergence of the axial-vector current.

\section{CONCLUSIONS}

We have shown in this work how the heat-kernel asymptotic expansion for theories at zero temperature can be generalized to incorporate temperature effects. In fact, we have shown that if we try to keep a close analogy with the zero-temperature case, all we can do is write a series expansion for the heat kernel, but this time with coefficients that, besides their $\beta$ dependence, also depend on the regularization parameter $\tau$, but in such a way that, for a fixed $\beta$, these "generalized coefficients" do not admit a power-series expansion at $\sqrt{\tau} / \beta=0$. We obtained a general expression for the $a_{m}(\sqrt{\tau} / \beta)$ and computed explicitly two of them associated with an operator of physical relevance.

We also observed that the dependence on these parameters is through the interesting ratio $\sqrt{\tau} / \beta$. This result reflects the fact that the regularization limit $\tau \rightarrow 0$ coincides precisely with the zero-temperature limit $\beta \rightarrow \infty$. Since anomalies can be calculated from Seeley's coefficients in the limit $\tau \rightarrow 0$, our result also provides a transparent way of confirming the well-known temperature independence of the anomalies. The physical interpretation for this fact is that the chiral anomaly is an ultraviolet kind of divergence (its origin can be viewed as due to the products of operators at the same point of space time), while temperature effects are sensible only near the mass shell. Some of these results were illustrated in our discussion of the axial model.

As a final remark, it is worth mentioning that, since the Casimir effect in theories at zero temperature arises from imposing spatial boundary conditions similar to those present in the time variable for theories at finite temperature, we think our results may be useful for this kind of calculation too.

\section{ACKNOWLEDGMENTS}

We would like to thank C. Sigaud for discussions on the Appendix. One of us (C.F.) thanks C. Wotzasek for helpful discussions, M. Asorey and J. L. Alonso for a careful reading of the manuscript and Departamento de Física Teorica, Zaragoza, for hospitality. This work was supported in part by CNPq and FAPERJ (Brazilian Agencies).

\section{APPENDIX}

Here, we shall prove (3.11) or, in the other words, we shall evaluate the series

$$
S=\sum_{n=-\infty}^{+\infty}\left[\gamma\left(n+\frac{1}{2}\right)^{2}\right]^{p} e^{-\gamma(n+1 / 2)^{2}}
$$

where $p$ is a non-negative integer. This series appears in the calculation of the heat kernel at finite temperature for Fermi fields. In fact, we are interested in transforming this slowly convergent series into a rapid one. This could be achieved in an elegant way with the aid of the Riemann $\zeta$ function (as well as the generalized Riemann $\zeta$ function). However, we propose an easier method based directly on Cauchy's theorem. Let us, then, con- 
sider the complex integral

$$
I=\oint_{C} \frac{\left(\gamma z^{2}\right)^{p} e^{-\gamma z^{2}}}{e^{2 \pi i z}+1} d z
$$

whose poles of the integrand lie at

$$
z=n+\frac{1}{2}, \quad n=0, \pm 1, \pm 2, \ldots .
$$

Choosing the contour $C$ conveniently in order to pick up the contribution of all poles (as in Fig. 1), the residue theorem leads to

$$
\begin{aligned}
I & =2 \pi i \text { i residues } \\
& =-\sum_{n=-\infty}^{+\infty}\left[\gamma\left(n+\frac{1}{2}\right)^{2}\right]^{p} e^{-\gamma(n+1 / 2)^{2}}=-S .
\end{aligned}
$$

Having established our main idea, let us proceed and perform the integration over the complex variable $z$. In Fig. 1, the contour $C$ is such that the limit $x_{0} \rightarrow \infty$ is taken in order to include all the poles (A3). Since $p$ is a fixed finite integer, the integral over the paths $C_{2}$ and $C_{4}$ vanish as $x_{0} \rightarrow \infty$.

To calculate the integral over $C_{1}$ we expand the denominator of the integrand,

$$
\frac{1}{e^{2 \pi i z}+1}=\sum_{n=0}^{\infty}(-1)^{n} e^{2 \pi i n z},
$$

where $z=x+i y_{0}, y_{0}>0$ and we can make $e^{2 \pi i z}$ as small as we want, so

$$
\begin{aligned}
I_{1}=\int_{-\infty+i y_{0}}^{+\infty+i y_{0}}\left[\gamma z^{2}\right]^{p} e^{-\gamma z^{2}} \sum_{n=0}^{\infty}(-1)^{n} e^{2 \pi i n z} d z \\
=\frac{-1}{\sqrt{\gamma}} \sum_{n=0}^{\infty}(-1)^{n} e^{-\pi^{2} n^{2} / \gamma} \int_{-\infty+i y_{0}}^{+\infty+i y_{0}}\left[z^{\prime}+\frac{i n \pi}{\sqrt{\gamma}}\right)^{2 p} \\
\times e^{-{z^{\prime}}^{2}} d z^{\prime},
\end{aligned}
$$

where $z^{\prime}=\sqrt{\gamma}(z-i n \pi / \gamma)$. Expanding the binomial

$$
\left(z^{\prime}+\frac{i n \pi}{\sqrt{\gamma}}\right)^{2 p}=\sum_{l=0}^{2 p} z^{(2 p-l)}\left(\frac{i n \pi}{\sqrt{\gamma}}\right)^{l} \frac{(2 p) !}{(2 p-l) ! l !},
$$

using the formula

$$
\int_{-\infty+i y_{0}}^{+\infty+i y_{0}} z^{\prime(2 p-2 l)} e^{-z^{\prime 2}} d z^{\prime}=\frac{(2 p-2 l-1) ! !}{2^{p-l}} \sqrt{\pi},
$$

where $(2 p-2 l-1) ! !=1 \times 3 \times 5 \cdots \times(2 p-2 l-1)$, and the fact that only even powers of $z^{\prime}$ contribute, we find

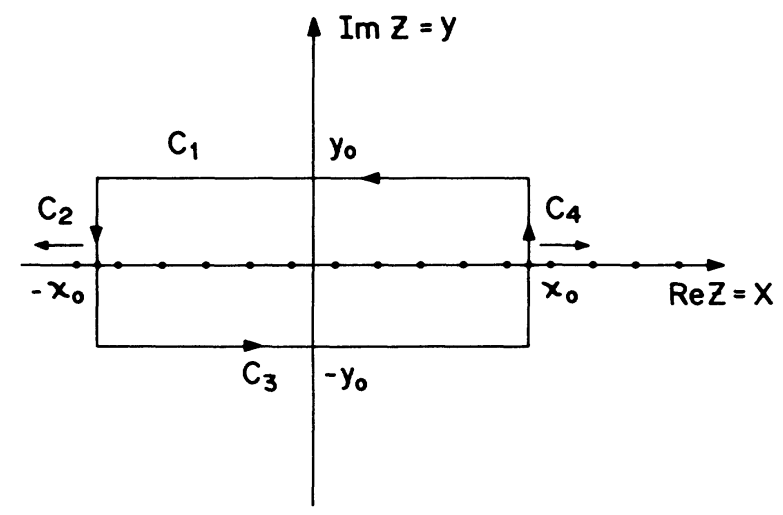

FIG. 1. A convenient contour which encloses all poles in the complex plane.

$$
I_{1}=-\left(\frac{\pi}{\gamma}\right)^{1 / 2} \sum_{n=0}^{\infty} e^{-\pi^{2} n^{2} / \gamma} \sum_{l=0}^{p}\left(\frac{i n \pi}{\sqrt{\gamma}}\right)^{2 l} \bar{C}_{l}^{p},
$$

where

$$
\bar{C}_{l}^{p}=\frac{(2 p) !}{(2 l) !(p-l) ! 2^{2 p-2 l}} .
$$

A similar calculation can be done for $I_{3}$ (corresponding to the contour $C_{3}$ ), but now, since $z=x-i y_{0}$, instead of (A5), we must expand the denominator of the integrand in (A2) as

$$
\begin{aligned}
\frac{1}{e^{2 \pi i z}+1}=\frac{e^{-2 \pi i z}}{e^{-2 \pi i z}+1} & =e^{-2 \pi i z} \sum_{n=0}^{\infty}(-1)^{n} e^{-2 \pi i n z} \\
& =\sum_{n=0}^{\infty}(-1)^{n} e^{-2 \pi i(n+1) z} .
\end{aligned}
$$

Hence, we find

$$
I_{3}=-\left(\frac{\pi}{\gamma}\right)^{1 / 2} \sum_{n=0}^{\infty}(-1)^{n} e^{-\pi^{2} n^{2} / \gamma} \sum_{l=0}^{p}\left(\frac{i n \pi}{\sqrt{\gamma}}\right)^{2 l} \bar{C}_{l}^{p} .
$$

Equating now

$$
S=-I=-I_{1}-I_{3},
$$

we finally obtain

$$
\begin{aligned}
S= & \left(\frac{\pi}{\gamma}\right]^{1 / 2} \frac{(2 p-1) ! !}{2 p} \\
& \times\left[1+2 \sum_{n=1}^{\infty}(-1)^{n} e^{-\pi^{2} n^{2} / \gamma} \sum_{l=0}^{p}\left[\frac{-\eta^{2} \pi^{2}}{\gamma}\right]^{l} C p\right],
\end{aligned}
$$

where $C P=\bar{C}_{l}^{p} / C_{0}^{p}$, which completes the proof of (3.11).
[1] L. Dolan and R. Jackiw, Phys. Rev. D 9, 3320 (1974).

[2] M. Reuter and W. Dittrich, Phys. Rev. D 32, 513 (1985).

[3] K. Fujikawa, Phys. Rev. Lett. 42, 1195 (1979); Phys. Rev. D 21, 2848 (1980).

[4] J. S. Dowker and R. Critchley, Phys. Rev. D 13, 3224 (1976); S. W. Hawking, Commun. Math. Phys. 55, 133 (1977); J. S. Dowker, J. Phys. A 11, 34 (1978); N. K. Niel- sen, M. T. Grisaru, H. Romer, and P. Van Nieuwenhuizen, Nucl. Phys. B140, 477 (1978).

[5] A. P. Balachandran, G. Marmo, V. P. Nair, and C. G. Trahern, Phys. Rev. D 25, 2713 (1982); M. Reuter, ibid. 31, 1374 (1985).

[6] R. E. Gamboa-Saraví, M. A. Muschietti, F. A. Schaposnik, and J. E. Solomin, Ann. Phys. (N.Y.) 157, 361 
(1983).

[7] This formalism was considered by R. Feynman and developed by T. Matsubara, Prog. Theor. Phys. 14, 351 (1955). See also C. Bernard, Phys. Rev. D 9, 3312 (1974).

[8] R. T. Seeley, Am. Math. Soc. Proc. Symp. Pure Math 10, 288 (1967).

[9] K. Rothe and I. O. Stamatescu, Ann. Phys. (N.Y.) 95, 202 (1975).

[10] R. Banerjee, Z. Phys. C 25, 251 (1984); A. Das and C. R. Hagen, Phys. Rev. D 32, 2024 (1985); C. Farina and A. Vaidya, ibid. 32, 2243 (1985).

[11] J. Barcelos-Neto, C. Farina, and A. Vaidya, Nuovo Cimento B96, 185 (1986).

[12] B. S. DeWitt, in Space-Time Approach to Quantum Field Theory, Proceedings of Les Houches Summer School, Les Houches, France, 1984, edited by B. S. DeWitt and R. Stora, Les Houches Summer School Proceedings Vol. XL
(North-Holland, Amsterdam, 1984).

[13] J. Schwinger, Phys. Rev. 82, 664 (1951).

[14] R. Nepomechie, Phys. Rev. D 31, 3291 (1985).

[15] M. de Souza Alves, C. Farina, and C. Wotzasek, Phys. Rev. D 43, 4145 (1991).

[16] R. J. Rivers, in Path Integral Methods in Quantum Field Theory, edited by P. V. Landshoff, D. Nelson, D. W. Scia$\mathrm{ma}$, and $\mathrm{S}$. Weinberg, Cambridge Monographs on Mathematical Physics (Cambridge University Press, Cambridge, England, 1987).

[17] B. Davies, Integral Transforms and their Applications (Springer-Verlag, New York, 1978); G. G. MacFarlane, Philos. Mag. 40, 188 (1949).

[18] Our conventions are $g^{00}=1=-g^{11} ; \quad\left(\gamma^{0}\right)^{\dagger}=\gamma^{0}, \quad\left(\gamma^{1}\right)^{\dagger}$ $=-\gamma^{1},\left(\gamma^{5}\right)^{\dagger}=\gamma^{5}=\gamma^{0} \gamma^{1} ;\left(\gamma^{0}\right)^{2}=1=\left(\gamma^{5}\right)^{2} ;\left(\gamma^{1}\right)^{2}=-1$.

[19] C. Wotzasek, C. Farina, and A. Vaidya, Nuovo Cimento B104, 185 (1989). 